\title{
Numerical Reconstruction of Spring-Mass System from Two Nondisjoint Spectra
}

\author{
Hubert Pickmann, ${ }^{1}$ Juan C. Egaña, ${ }^{2}$ and Ricardo L. Soto ${ }^{2}$ \\ ${ }^{1}$ Departamento de Matemática, Universidad de Tarapacá, 1010069 Arica, Chile \\ ${ }^{2}$ Departamento de Matemáticas, Universidad Católica del Norte, 1270709 Antofagasta, Chile
}

Correspondence should be addressed to Ricardo L. Soto; rsoto@ucn.cl

Received 13 May 2015; Accepted 10 June 2015

Academic Editor: Filippo Ubertini

Copyright (c) 2015 Hubert Pickmann et al. This is an open access article distributed under the Creative Commons Attribution License, which permits unrestricted use, distribution, and reproduction in any medium, provided the original work is properly cited.

A new numerical procedure is presented to reconstruct a fixed-free spring-mass system from two auxiliary spectra, which are nondisjoint. The method is a modification of the fast orthogonal reduction algorithm, which is less computationally expensive than others in the literature. Numerical results are obtained, showing the accuracy of the algorithm.

\section{Introduction}

The inverse problems in structures vibration look for determining or estimating the physical properties of a system in vibration (mass density, elastic constants, etc.) from a known dynamic behavior (natural frequencies, electric flux, tension, etc.) (see [1-4]).

The model used, which has generated much interest in the literature, as a prototype of structure, is a nonuniform thin rod with one end fixed to a surface (see [2-5]), whose discrete model is a spring-mass system, which consists of $n$ mass $m_{i}>0$, associated with the masses of each element of the rod and connected by $n$ springs with rigidity constants $k_{i}>0$ corresponding to the rigidity of each one of these elements (Figure 1).

A spring-mass system in free and longitudinal vibration is governed by a generalized eigenvalue problem of the form (see $[1-4]$ )

$$
(K-\lambda M) u=0, \quad \lambda=\omega^{2},
$$

where

$$
K=\left(\begin{array}{ccccc}
k_{1}+k_{2} & -k_{2} & & & \\
-k_{2} & k_{2}+k_{3} & -k_{3} & & \\
& \ddots & \ddots & \ddots & \\
& & -k_{n-1} & k_{n-1}+k_{n} & -k_{n} \\
& & & -k_{n} & k_{n}
\end{array}\right)
$$

and $M=\operatorname{diag}\left\{m_{1}, m_{2}, \ldots, m_{n}\right\}$ are the stiffness matrix and the mass matrix, respectively, and $u$ is the displacement vector. In this system, the eigenvalues $\lambda_{i}, i=1,2, \ldots, n$, of (1) are related to the natural frequencies $\omega_{i}$ and the eigenvectors $u^{(i)}=\left(u_{1}^{(i)}, u_{2}^{(i)}, \ldots, u_{n}^{(i)}\right)^{T}$ represent the vibration modes of the system. The spring-mass system is denoted by $(M, K)$.

It is known (see [4]) that the matrices $M$ and $K$ can be uniquely reconstructed if the following information is given: the eigenvalues $\left(\lambda_{i}\right)_{1}^{n}$ of the original system $(M, K)$, the eigenvalues $\left(\mu_{i}\right)_{1}^{n-1}$ of the auxiliary system $(\bar{M}, \bar{K})$, corresponding to the original system whose last mass is fixed (Figure 2), and an additional factor, for example, the total mass of the system $m_{T}=\sum_{i=1}^{n} m_{i}$.

The structural properties of the matrices $M$ and $K$ allow us to reduce the generalized eigenvalue equation (1) to the standard form (see [1-4])

$$
\begin{aligned}
\left(J-\lambda_{i} I\right) v^{(i)} & =0, \\
J & =B^{-1} K B^{-1}, \\
v^{(i)} & =B u^{(i)}, \\
B & =\operatorname{diag}\left\{m_{1}^{1 / 2}, m_{2}^{1 / 2}, \ldots, m_{r}^{1 / 2}\right\},
\end{aligned}
$$

where the Jacobi matrix $J$ is tridiagonal symmetric positive definite, with the same eigenvalues $\left(\lambda_{i}\right)_{1}^{n}$ of the system $(M, K)$, 


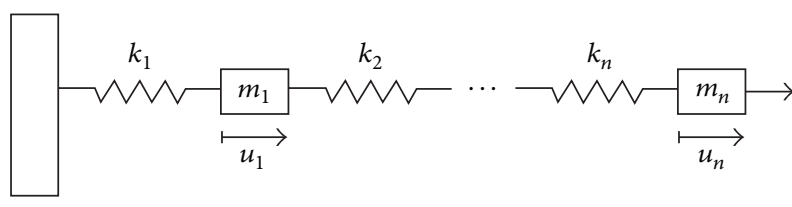

FIGURE 1: Fixed-free spring-mass system.

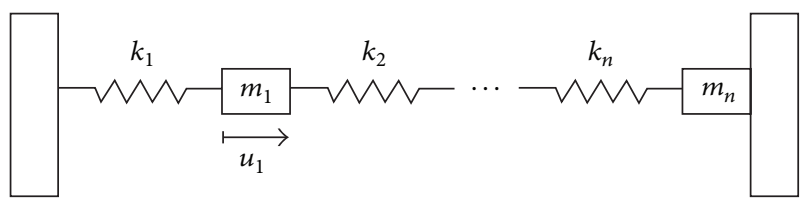

FIGURE 2: Fixed-fixed spring-mass system.

which are real, positive, and distinct. Therefore, a fundamental step to determine the system $(M, K)$ is to reconstruct the matrix $J$. Without loss of generality, we assume that $J$ is of the following form:

$$
J=\left(\begin{array}{ccccc}
a_{1} & b_{1} & & & \\
b_{1} & a_{2} & b_{2} & & \\
& \ddots & \ddots & \ddots & \\
& & b_{n-2} & a_{n-1} & b_{n-1} \\
& & & b_{n-1} & a_{n}
\end{array}\right), \quad a_{i}, b_{i}>0 .
$$

In [6], stable numerical procedures to reconstruct the Jacobi matrix $J$ are discussed. This reconstruction uses as initial spectral information the eigenvalues $\left(\lambda_{i}\right)_{1}^{n}$ of $J$ and the eigenvalues $\left(\mu_{i}\right)_{1}^{n-1}$ of the matrix $\bar{J}$, which is obtained by deleting the last row and last column of $J$. A fundamental property in these procedures is the interlacing property (see $[1,4,6])$

$$
\lambda_{1}<\mu_{1}<\lambda_{2}<\cdots<\mu_{n-1}<\lambda_{n}
$$

which is a necessary and sufficient condition for the existence of a physically real system and for constructing $J$ as well.

In $[7,8]$ the authors generalize the reconstruction of the system $(M, K)$ by using the interlaced spectrum corresponding to an auxiliary system that consists in fixing any mass of the system $(M, K)$, other than the extreme masses (Figure 3 ).

Clearly, if the auxiliary system $(\bar{M}, \bar{K})$ is the system with its $(r+1)$ th mass, $1 \leq r \leq n-2$, being fixed, then $(\bar{M}, \bar{K})$ is uncoupled in two auxiliary spring-mass systems, $\left(\bar{M}_{r}, \bar{K}_{r}\right)$ and $\left(\bar{M}_{p}, \bar{K}_{p}\right)$, with natural frequencies $\left(\gamma_{i}\right)_{1}^{r}$ and $\left(\eta_{i}\right)_{1}^{p}$, respectively, where $p=n-r-1$. The structural properties of the matrices $\bar{M}_{r}, \bar{K}_{r}, \bar{M}_{p}$, and $\bar{K}_{p}$ allow us to partition $J$ as

$$
J=\left[\begin{array}{ccc}
J_{r} & b_{r} e_{r} & \mathbf{0} \\
b_{r} e_{r}^{T} & a_{r+1} & b_{r+1} e_{1}^{T} \\
\mathbf{0} & b_{r+1} e_{1} & J_{p}
\end{array}\right],
$$

where the submatrices $J_{r}=B_{r}^{-1} K_{r} B_{r}^{-1}$ and $J_{p}=$ $B_{p}^{-1} K_{p} B_{p}^{-1}$, with $B_{r}=\operatorname{diag}\left\{m_{1}^{1 / 2}, m_{2}^{1 / 2}, \ldots, m_{r}^{1 / 2}\right\}$ and
$B_{p}=\operatorname{diag}\left\{m_{r+2}^{1 / 2}, m_{r+3}^{1 / 2}, \ldots, m_{n}^{1 / 2}\right\}$, are related to the systems $\left(\bar{M}_{r}, \bar{K}_{r}\right)$ and $\left(\bar{M}_{p}, \bar{K}_{p}\right)$, respectively. As the system $(M, K)$ can be reconstructed from the matrix $J$, it is enough to reconstruct $J$ from the sets $\left(\lambda_{i}\right)_{1}^{n}$ and $\left(\mu_{i}\right)_{1}^{n-1}=\left(\gamma_{i}\right)_{1}^{r} \cup\left(\eta_{i}\right)_{1}^{p}$ to obtain $(M, K)$. Thus, the reconstruction of the system $(M, K)$ is reduced to the following problem.

Problem 1. Given the sequence of real numbers $\left(\lambda_{i}\right)_{1}^{n}$, $\left(\mu_{i}\right)_{1}^{n-1}=\left(\gamma_{i}\right)_{1}^{r} \cup\left(\eta_{i}\right)_{1}^{p}$ satisfying the interlacing property

$$
\lambda_{i} \leq \mu_{i} \leq \lambda_{i+1}, \quad 1 \leq i \leq n-1,
$$

reconstruct the matrix $J$ in (6), such that

$$
\begin{aligned}
\sigma(J) & =\left(\lambda_{i}\right)_{1}^{n}, \\
\sigma\left(J_{r}\right) & =\left(\gamma_{i}\right)_{1}^{r}, \\
\sigma\left(J_{p}\right) & =\left(\eta_{i}\right)_{1}^{p}, \\
p & =n-r-1 .
\end{aligned}
$$

In this problem two cases arise: in the first one, all natural frequencies $\left(\gamma_{i}\right)_{1}^{r}$ and $\left(\eta_{i}\right)_{1}^{p}$ are distinct; that is, $\left(\gamma_{i}\right)_{1}^{r} \cap\left(\eta_{i}\right)_{1}^{p}=\emptyset$. In terms of the $J$ matrix, the meaning is that no eigenvector $v^{(i)}$ of $J$ has a node in its coordinate $v_{m+1}^{(i)}$; that is, $v_{m+1}^{(i)} \neq$ $0, i=1, \ldots, n$. In this case, the reconstruction is unique. In the second case, one or more natural frequencies $\left(\gamma_{i}\right)_{1}^{r}$ and $\left(\eta_{i}\right)_{1}^{p}$, are identical. The meaning of this situation is that some eigenvector of $J$, let us say $v^{(\ell)}$, has a node in $v_{m+1}^{(\ell)}$; that is, $v_{m+1}^{(\ell)}=0$. In this case, $\left(\gamma_{i}\right)_{1}^{r} \cap\left(\eta_{i}\right)_{1}^{p} \cap\left(\lambda_{i}\right)_{1}^{n} \neq \emptyset$, and a family of isospectral matrices is obtained.

In [7], the authors study the first case; that is, they reconstruct the system $(M, K)$, using a modification of the fast orthogonal reduction method, when the auxiliary spectra are separated. In the next section we study the second case, using the same method, and thus the problem is completely solved. This method is less computationally expensive than others in literature [8].

\section{Reconstructing the System from Nondisjoint Spectra}

We denote by $P_{n}(\lambda), Q_{r}(\lambda)$, and $S_{p}(\lambda)$ the characteristic polynomials of the matrices $J, J_{r}$, and $J_{p}$, respectively; that is,

$$
\begin{aligned}
P_{n}(\lambda) & =\prod_{i=1}^{n}\left(\lambda-\lambda_{i}\right) ; \\
Q_{r}(\lambda) & =\prod_{i=1}^{r}\left(\lambda-\gamma_{i}\right) ; \\
S_{p}(\lambda) & =\prod_{i=1}^{p}\left(\lambda-\eta_{i}\right) .
\end{aligned}
$$




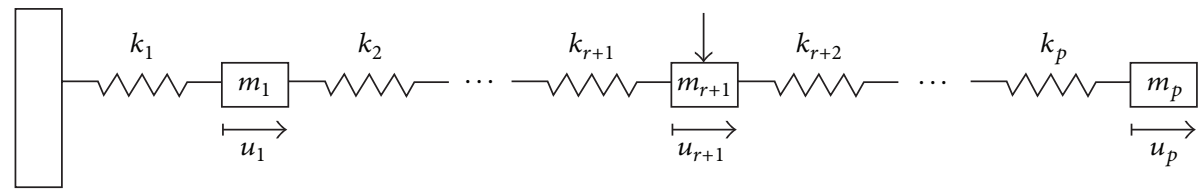

FIGURE 3: Spring-mass system with a fixed interior mass.

We define the vectors

$$
\begin{gathered}
u_{r}=\left(u_{r, r}^{(r)}, u_{r, r}^{(r-1)}, \ldots, u_{r, r}^{(1)}\right)^{T}, \\
w_{p}=\left(w_{p, 1}^{(1)}, w_{p, 1}^{(2)}, \ldots, w_{p, 1}^{(p)}\right)^{T},
\end{gathered}
$$

corresponding, respectively, to the last and the first row of the matrices of eigenvectors of $J_{r}$ and $J_{p}$. We also define the diagonal matrices $\Delta_{r}=\operatorname{diag}\left\{\gamma_{r}, \gamma_{r-1}, \ldots, \gamma_{1}\right\}$ and $\Delta_{p}=$ $\operatorname{diag}\left\{\eta_{1}, \eta_{2}, \ldots, \eta_{p}\right\}$.

Theorem 2. Let the real numbers $\left(\lambda_{i}\right)_{1}^{n}$ and $\left(\mu_{i}\right)_{1}^{n-1}=\left(\gamma_{i}\right)_{1}^{r} \cup$ $\left(\eta_{i}\right)_{1}^{p}, p=n-r-1$ be given, satisfying the interlacing property (7); that is, $\left(\gamma_{i}\right)_{1}^{r} \cap\left(\eta_{i}\right)_{1}^{p} \cap\left(\lambda_{i}\right)_{1}^{n} \neq \emptyset$. Then there exists an isospectral family of $n \times n$ matrices $J(\alpha), \alpha \in(0, \pi / 2)$, of the form (6) such that $\sigma(J(\alpha))=\left(\lambda_{i}\right)_{1}^{n}, \sigma\left(J_{r}(\alpha)\right)=\left(\gamma_{i}\right)_{1}^{r}$, and $\sigma\left(J_{p}(\alpha)\right)=\left(\eta_{i}\right)_{1}^{p}$.

Proof. We suppose that there is a pair $\gamma_{j}, \eta_{k}$ of frequencies such that $\gamma_{j}=\eta_{k}=\lambda_{m}$, where $m=j+k$. From the expansion of $\operatorname{det}\left(\lambda I_{n}-J_{n}\right)$, throughout its $(r+1)$ th row, we find that

$$
\begin{aligned}
P_{n}(\lambda)= & \left(\lambda-a_{r+1}\right) Q_{r}(\lambda) S_{p}(\lambda)-b_{r}^{2} Q_{r \backslash r}(\lambda) S_{p}(\lambda) \\
& -b_{r+1}^{2} Q_{r}(\lambda) S_{p \backslash 1}(\lambda) \\
= & -b_{r+1}^{2} Q_{r}(\lambda) S_{p \backslash 1}(\lambda) \\
& +S_{p}(\lambda)\left[\left(\lambda-a_{r+1}\right) Q_{r}(\lambda)-b_{r}^{2} Q_{r \backslash r}(\lambda)\right],
\end{aligned}
$$

where $S_{p \backslash 1}(\lambda)$ and $Q_{r \backslash r}(\lambda)$ are the characteristic polynomials of $J_{p}$ and $J_{r}$ after we delete its first row and column, and $r$ th row and column, respectively.

On the other hand, if we denote by $Q_{r+1}(\lambda)$ the characteristic polynomial of the principal submatrix obtained from $J_{r}$ by adding $(r+1)$ th row and column, we have that

$$
Q_{r+1}(\lambda)=\left(\lambda-a_{r+1}\right) Q_{r}(\lambda)-b_{r}^{2} Q_{r \backslash r}(\lambda) .
$$

Thus, (11) is

$$
P_{n}(\lambda)=S_{p}(\lambda) Q_{r+1}(\lambda)-b_{r+1}^{2} Q_{r}(\lambda) S_{p \backslash 1}(\lambda) .
$$

Analogously, (11) can be written as

$$
\begin{aligned}
P_{n}(\lambda)= & -b_{r}^{2} Q_{r \backslash r}(\lambda) S_{p}(\lambda) \\
& +Q_{r}(\lambda)\left[\left(\lambda-a_{r+1}\right) S_{p}(\lambda)-b_{r+1}^{2} S_{p \backslash 1}(\lambda)\right] .
\end{aligned}
$$

Now, if we denote by $S_{p+1}(\lambda)$ the characteristic polynomial of the principal submatrix obtained from $J_{p}$ by adding a row and column above $J_{p}$, we have

$$
S_{p+1}(\lambda)=\left(\lambda-a_{r+1}\right) S_{p}(\lambda)-b_{r+1}^{2} S_{p \backslash 1}(\lambda) .
$$

Then,

$$
P_{n}(\lambda)=Q_{r}(\lambda) S_{p+1}(\lambda)-b_{r}^{2} Q_{r \backslash r}(\lambda) S_{p}(\lambda) .
$$

From (12) we have

$$
Q_{r+1}\left(\gamma_{j}\right)=-b_{r}^{2} Q_{r \backslash r}\left(\gamma_{j}\right),
$$

and from (15) we have

$$
S_{p+1}\left(\eta_{k}\right)=-b_{r+1}^{2} S_{p \backslash 1}\left(\eta_{k}\right) .
$$

Since the polynomials $P_{n}(\lambda), Q_{r}(\lambda)$, and $S_{p}(\lambda)$ in (9) have common factors $\lambda-\lambda_{m} \equiv \lambda-\eta_{k} \equiv \lambda-\gamma_{j}$, (13) and (16) are

$$
\begin{aligned}
& P_{n}^{\prime}\left(\lambda_{m}\right)=S_{p}^{\prime}\left(\eta_{k}\right) Q_{r+1}\left(\gamma_{j}\right)-b_{r+1}^{2} Q_{r}^{\prime}\left(\gamma_{j}\right) S_{p \backslash 1}\left(\eta_{k}\right), \\
& P_{n}^{\prime}\left(\lambda_{m}\right)=Q_{r}^{\prime}\left(\gamma_{j}\right) S_{p+1}\left(\eta_{k}\right)-b_{r}^{2} Q_{r \backslash r}\left(\gamma_{j}\right) S_{p}^{\prime}\left(\eta_{k}\right),
\end{aligned}
$$

respectively, where

$$
\begin{aligned}
Q_{r}^{\prime}\left(\gamma_{i}\right) & =\prod_{\substack{j=1 \\
j \neq i}}^{r}\left(\gamma_{i}-\gamma_{j}\right), \\
S_{p}^{\prime}\left(\eta_{i}\right) & =\prod_{\substack{j=1 \\
j \neq i}}^{p}\left(\eta_{i}-\eta_{j}\right), \\
P_{n}^{\prime}\left(\lambda_{m}\right) & =\prod_{\substack{i=1 \\
i \neq m}}^{n}\left(\lambda_{m}-\lambda_{i}\right) .
\end{aligned}
$$

Replacing (17) and (18) in these last two equations, we obtain

$$
\begin{aligned}
P_{n}^{\prime}\left(\lambda_{m}\right)= & -b_{r}^{2} Q_{r \backslash r}\left(\gamma_{j}\right) S_{p}^{\prime}\left(\eta_{k}\right) \\
& -b_{r+1}^{2} Q_{r}^{\prime}\left(\gamma_{j}\right) S_{p \backslash 1}\left(\eta_{k}\right)
\end{aligned}
$$

and dividing (22) by $S_{p}^{\prime}\left(\eta_{k}\right) Q_{r}^{\prime}\left(\gamma_{j}\right)$, we get

$$
\begin{aligned}
\frac{P_{n}^{\prime}\left(\lambda_{m}\right)}{S_{p}^{\prime}\left(\eta_{k}\right) Q_{r}^{\prime}\left(\gamma_{j}\right)}= & -b_{r}^{2}\left[\frac{Q_{r \backslash r}\left(\gamma_{j}\right)}{Q_{r}^{\prime}\left(\gamma_{j}\right)}\right] \\
& -b_{r+1}^{2}\left[\frac{S_{p \backslash 1}\left(\eta_{k}\right)}{S_{p}^{\prime}\left(\eta_{k}\right)}\right] .
\end{aligned}
$$

It is known that if $U_{r}=\left[u_{r}^{(1)} u_{r}^{(2)} \cdots u_{r}^{(r)}\right]$ is the orthogonal matrix of eigenvectors of $J_{r}$, then $U_{r}^{T}\left(\lambda I_{r}-J_{r}\right) U_{r}=\lambda I_{r}-\Lambda_{r}$, and we have

$$
\left(\lambda I_{r}-J_{r}\right)^{-1}=U_{r}\left(\lambda I_{r}-\Lambda_{r}\right) U_{r}^{T}
$$


The left side in (24) is

$$
\begin{aligned}
\left(\lambda I_{r}-J_{r}\right)^{-1} & =\frac{1}{Q_{r}(\lambda)} \cdot \operatorname{adj}\left(\lambda I_{r}-J_{r}\right) \\
& =\frac{1}{Q_{r}(\lambda)} \cdot\left[\begin{array}{cccc}
* & * & * & * \\
* & * & * & * \\
\vdots & \vdots & \vdots & \vdots \\
* & * & * & Q_{r \backslash r}(\lambda)
\end{array}\right]
\end{aligned}
$$

while the right side is

$$
U_{r}\left(\lambda I_{r}-\Lambda_{r}\right) U_{r}^{T}=\left[\frac{u_{r}^{(1)}}{\lambda-\gamma_{1}} \frac{u_{r}^{(2)}}{\lambda-\gamma_{2}} \cdots \frac{u_{r}^{(r)}}{\lambda-\gamma_{r}}\right] U_{r}^{T} .
$$

Comparing the entries in position $(r, r)$ in both sides in (24) we find that

$$
\frac{Q_{r \backslash r}(\lambda)}{Q_{r}(\lambda)}=\sum_{i=1}^{r} \frac{\left[u_{r, r}^{(i)}\right]^{2}}{\lambda-\gamma_{i}} .
$$

Taking the limit when $\lambda$ tends to $\gamma_{i}$, we obtain

$$
\frac{Q_{r \backslash r}\left(\gamma_{j}\right)}{Q_{r}^{\prime}\left(\gamma_{j}\right)}=\left[u_{r, r}^{(j)}\right]^{2} \text {. }
$$

Analogously, we can obtain

$$
\frac{S_{p \backslash 1}\left(\eta_{k}\right)}{S_{p}^{\prime}\left(\eta_{k}\right)}=\left[w_{p, 1}^{(k)}\right]^{2} .
$$

Then, by replacing (28) and (29) in (23), we get

$$
-\left(\frac{P_{n}^{\prime}\left(\lambda_{m}\right)}{S_{p}^{\prime}\left(\eta_{k}\right) Q_{r}^{\prime}\left(\gamma_{j}\right)}\right)=b_{r}^{2}\left[u_{r, r}^{(j)}\right]^{2}+b_{r+1}^{2}\left[w_{p, 1}^{(k)}\right]^{2} \text {. }
$$

For $\alpha \in(0, \pi / 2)$ we can define

$$
\begin{aligned}
b_{r}^{2}(\alpha)\left[u_{r, r}^{(j)}(\alpha)\right]^{2} & =-\left(\frac{P_{n}^{\prime}\left(\lambda_{m}\right)}{S_{p}^{\prime}\left(\eta_{k}\right) Q_{r}^{\prime}\left(\gamma_{j}\right)}\right) \cos ^{2} \alpha, \\
b_{r+1}^{2}(\alpha)\left[w_{p, 1}^{(k)}(\alpha)\right]^{2} & =-\left(\frac{P_{n}^{\prime}\left(\lambda_{m}\right)}{S_{p}^{\prime}\left(\eta_{k}\right) Q_{r}^{\prime}\left(\gamma_{j}\right)}\right) \sin ^{2} \alpha .
\end{aligned}
$$

Thus, given that

$$
\begin{aligned}
b_{r}^{2}(\alpha)= & -\left(\frac{P_{n}^{\prime}\left(\gamma_{j}\right)}{S_{p}^{\prime}\left(\gamma_{j}\right) Q_{r}^{\prime}\left(\gamma_{j}\right)}\right) \cos ^{2} \alpha \\
& +\sum_{\substack{i=1 \\
i \neq j}}^{r}-\left(\frac{P_{n}\left(\gamma_{i}\right)}{S_{p}\left(\gamma_{i}\right) Q_{r}^{\prime}\left(\gamma_{i}\right)}\right), \\
b_{r+1}^{2}(\alpha)= & -\left(\frac{P_{n}^{\prime}\left(\eta_{k}\right)}{S_{p}^{\prime}\left(\eta_{k}\right) Q_{r}^{\prime}\left(\eta_{k}\right)}\right) \sin ^{2} \alpha \\
& +\sum_{\substack{i=1 \\
i \neq j}}^{p}\left(\frac{P_{n}\left(\eta_{i}\right)}{S_{p}^{\prime}\left(\eta_{i}\right) Q_{r}\left(\eta_{i}\right)}\right),
\end{aligned}
$$

(31) allow us to know $u_{r, r}^{(j)}(\alpha)$ and $w_{p, 1}^{(k)}(\alpha)$.
Subsequently, once the vectors $u_{r}(\alpha)$ and $w_{p}(\alpha)$ in (10) are known, we can form the $(n+1) \times(n+1)$ matrices:

$$
\begin{aligned}
& A_{r+1}(\alpha)=\left[\begin{array}{cc}
\Lambda_{r} & u_{r}(\alpha) \\
u_{r}^{T}(\alpha) & a_{0}^{r}
\end{array}\right], \\
& A_{p+1}(\alpha)=\left[\begin{array}{cc}
a_{0}^{p} & w_{r}^{T}(\alpha) \\
w_{r}(\alpha) & \Lambda_{p}
\end{array}\right],
\end{aligned}
$$

where the entries $a_{0}^{r}$ and $a_{0}^{p}$ are arbitrary real numbers. Then, we apply the Modified Fast Orthogonal Reduction Algorithm (see [9]) to orthogonally reduce the matrices $A_{r+1}(\alpha)$ and $A_{p+1}(\alpha)$ to their tridiagonal form, obtaining in this way the desired matrices $J_{r}(\alpha)$ and $J_{p}(\alpha)$. To do this, we first permute the arrowhead matrix $A_{r+1}(\alpha)$ by applying $P=\left[{ }_{1},{ }^{1}\right]$. We point out that similar relationships are analyzed by Jessup in [10].

Finally, considering that the diagonal entry $a_{r+1}$ of $J(\alpha)$ can be computed as

$$
a_{r+1}=\sum_{i=1}^{n} \lambda_{i}-\sum_{i=1}^{r} \gamma_{i}-\sum_{i=1}^{p} \eta_{i}
$$

and the codiagonal entries $b_{r}(\alpha)$ and $b_{r+1}(\alpha)$ can be computed from (32) and (33), respectively, the matrix $J(\alpha)$ of the form (6) is obtained completely, having a common eigenvalue with $J_{r}(\alpha)$ and $J_{p}(\alpha)$.

If we have more common eigenvalues, we can repeat the previous procedure for each pair of common eigenvalues. That is, if $s$ is the number of identical pairs $\gamma_{j_{q}} \equiv \eta_{k_{q}} \equiv \lambda_{m_{q}}$, $q=1, \ldots, s$, then we have

$$
\begin{aligned}
& b_{r}^{2}(\alpha)\left[u_{r, r}^{(i)}(\alpha)\right]^{2}=-\left(\frac{P_{n}\left(\gamma_{i}\right)}{S_{p}\left(\gamma_{i}\right) Q_{r}^{\prime}\left(\gamma_{i}\right)}\right) \\
& i^{*}=1,2, \ldots, r,
\end{aligned}
$$

$$
\begin{array}{r}
b_{r}^{2}(\alpha)\left[u_{r, r}^{\left(j_{q}\right)}(\alpha)\right]^{2}=-\left(\frac{P_{n}^{\prime}\left(\gamma_{j_{q}}\right)}{S_{p}^{\prime}\left(\gamma_{j_{q}}\right) Q_{r}^{\prime}\left(\gamma_{j_{q}}\right)}\right) \cos ^{2} \alpha, \\
q=1,2, \ldots, s,
\end{array}
$$$$
b_{r+1}^{2}(\alpha)\left[w_{p, 1}^{(i)}(\alpha)\right]^{2}=-\left(\frac{P_{n}\left(\eta_{i}\right)}{S_{p}^{\prime}\left(\eta_{i}\right) Q_{r}\left(\eta_{i}\right)}\right)
$$

$$
i^{*}=1,2, \ldots, p,
$$

$$
\begin{aligned}
& b_{r+1}^{2}(\alpha)\left[w_{p, 1}^{\left(k_{q}\right)}(\alpha)\right]^{2} \\
& \quad=-\left(\frac{P_{n}^{\prime}\left(\eta_{k_{q}}\right)}{S_{p}^{\prime}\left(\eta_{k_{q}}\right) Q_{r}^{\prime}\left(\eta_{k_{q}}\right)}\right) \sin ^{2} \alpha, \quad q=1,2, \ldots, s .
\end{aligned}
$$


Thus,

$$
\begin{aligned}
b_{r}^{2}(\alpha)= & \sum_{i=1}^{r^{*}}-\left(\frac{P_{n}\left(\gamma_{i}\right)}{S_{p}\left(\gamma_{i}\right) Q_{r}^{\prime}\left(\gamma_{i}\right)}\right) \\
& +\cos ^{2} \alpha \sum_{q=1}^{s}-\left(\frac{P_{n}^{\prime}\left(\gamma_{j_{q}}\right)}{S_{p}^{\prime}\left(\gamma_{j_{q}}\right) Q_{r}^{\prime}\left(\gamma_{j_{q}}\right)}\right), \\
b_{r+1}^{2}(\alpha)= & \sum_{i=1}^{p^{*}}-\left(\frac{P_{n}\left(\eta_{i}\right)}{S_{p}^{\prime}\left(\eta_{i}\right) Q_{r}\left(\eta_{i}\right)}\right) \\
& +\sin ^{2} \alpha \sum_{q=1}^{s}-\left(\frac{P_{n}^{\prime}\left(\eta_{k_{q}}\right)}{S_{p}^{\prime}\left(\eta_{k_{q}}\right) Q_{r}^{\prime}\left(\eta_{k_{q}}\right)}\right),
\end{aligned}
$$

where $(*)$ means that $s$ terms $i=j_{q}$ and $i=k_{q}$, $q=1, \ldots, s$, respectively, are omitted. Thus, we obtain an isospectral family of tridiagonal matrices that have identical eigenvalues.

\section{An Optimization Procedure to Find an Objective Jacobi Matrix}

In this section we want to find an objective matrix within a family of matrices. First, we observe that the construction procedure depends continuously on the parameter $\alpha$. Then, by means of an optimization procedure, we find an appropriate $\alpha$, so that the procedure reconstructs a matrix with a desired structure.

Theorem 3. Let $\widetilde{J}$ be a given symmetric tridiagonal matrix partitioned in the following form:

$$
\widetilde{J}=\left[\begin{array}{ccc}
\tilde{J}_{r} & \tilde{b}_{r} e_{r} & 0 \\
\tilde{b}_{r} e_{r}^{T} & \tilde{a}_{r+1} & \tilde{b}_{r+1} e_{1}^{T} \\
0 & \tilde{b}_{r+1} e_{1} & \tilde{J}_{p}
\end{array}\right],
$$

where $\sigma(\widetilde{J})=\left(\lambda_{i}\right)_{1}^{n}, \sigma\left(\widetilde{J}_{r}\right)=\left(\gamma_{i}\right)_{1}^{r}$, and $\sigma\left(\widetilde{J}_{p}\right)=\left(\eta_{i}\right)_{1}^{p}$, with $p=n-r-1$ and $\left(\gamma_{i}\right)_{1}^{r} \cap\left(\eta_{i}\right)_{1}^{p} \cap\left(\lambda_{i}\right)_{1}^{n} \neq \emptyset$. For $\varepsilon>0$ small enough the function, $F:[0+\varepsilon, \pi / 2-\varepsilon] \rightarrow \mathbb{R}$, defined by

$$
F(\alpha)=\|J(\alpha)-\widetilde{J}\|_{2}^{2}=\sum\left[j_{i k}(\alpha)-\widetilde{j}_{i k}\right]^{2},
$$

where the matrix $J(\alpha)$ is obtained by using the Modified Fast Orthogonal Reduction process, has a minimum in $[0+\varepsilon, \pi / 2-$ $\varepsilon]$.

Proof. Given $\alpha \in(0, \pi / 2)$, the Modified Fast Orthogonal Reduction process reconstructs a matrix $J(\alpha)$ of the form (38), from its eigenvalues. We show that all the entries of the matrix $J(\alpha)$ depend continuously on $\alpha$. In fact, expressions (37) are clear since $\cos (\alpha)$ and $\sin (\alpha)$ are not zero in $(0, \pi / 2)$. That is,

$$
\begin{array}{r}
b_{r}(\alpha) \neq 0, \\
b_{r+1}(\alpha) \neq 0 .
\end{array}
$$

The functions

$$
\begin{aligned}
& u_{r, r}^{(i)}(\alpha)=\sqrt{-\left(\frac{P_{n}\left(\gamma_{i}\right)}{S_{p}\left(\gamma_{i}\right) Q_{r}^{\prime}\left(\gamma_{i}\right)}\right) \frac{1}{b_{r}^{2}(\alpha)},} i^{*}=1,2, \ldots, r, \\
& u_{r, r}^{\left(j_{q}\right)}(\alpha)=\sqrt{-\left(\frac{P_{n}^{\prime}\left(\gamma_{j_{q}}\right)}{S_{p}^{\prime}\left(\gamma_{j_{q}}\right) Q_{r}^{\prime}\left(\gamma_{j_{q}}\right)}\right) \frac{\cos ^{2} \alpha}{b_{r}^{2}(\alpha)},} \\
& w_{p, 1}^{(i)}(\alpha)=\sqrt{-\left(\frac{P_{n}\left(\eta_{i}\right)}{S_{p}^{\prime}\left(\eta_{i}\right) Q_{r}\left(\eta_{i}\right)}\right) \frac{1}{b_{r+1}^{2}(\alpha)},}, \ldots, s, \\
& w_{p, 1}^{\left(k_{q}\right)}(\alpha)=\sqrt{-\left(\frac{P_{n}^{\prime}\left(\eta_{k_{q}}\right)}{S_{p}^{\prime}\left(\eta_{k_{q}}\right) Q_{r}^{\prime}\left(\eta_{k_{q}}\right)}\right) \frac{\sin ^{2} \alpha}{b_{r+1}^{2}(\alpha)},}, \ldots, p, \\
& q=1,2, \ldots, s,
\end{aligned}
$$

are continuous. Therefore, all the matrices

$$
\begin{aligned}
& A_{r+1}(\alpha)=\left[\begin{array}{ll}
\Lambda_{r}(\alpha) & u_{r}(\alpha) \\
u_{r}^{T}(\alpha) & a_{0}^{r}(\alpha)
\end{array}\right] \\
& A_{p+1}(\alpha)=\left[\begin{array}{ll}
a_{0}^{p}(\alpha) & w_{p}^{T}(\alpha) \\
w_{p}(\alpha) & \Lambda_{p}(\alpha)
\end{array}\right]
\end{aligned}
$$

have continuous entries. Since, in the Modified Fast Orthogonal Reduction process, the tridiagonalization matrices have rational entries with nonzero denominators, matrices $J_{r}(\alpha), J_{p}(\alpha)$, and, thus, $J(\alpha)$ depend continuously on $\alpha \epsilon$ $(0, \pi / 2)$.

Now, if $\alpha=0$ and $\left(\gamma_{i}\right)_{1}^{r} \cap\left(\eta_{i}\right)_{1}^{p} \cap\left(\lambda_{i}\right)_{1}^{n}=\left(v_{i}\right)_{1}^{p}$, then $b_{r+1}(\alpha)=0$. Afterwards, due to the discontinuity of $w_{p, 1}(\alpha)$, the reconstruction procedure is not completed. Analogously, when $\alpha=\pi / 2$ and $(\gamma)_{1}^{r} \cap(\eta)_{1}^{p} \cap(\lambda)_{1}^{n}=(\gamma)_{1}^{r}$, we have $b_{r}(\alpha)=0$, where again a discontinuity of $u_{r, r}(\alpha)$ is produced. Therefore, by affixing, $0<\varepsilon \ll 1$, the function

$$
F(\alpha)=\|J(\alpha)-\widetilde{J}\|_{2}^{2}=\sum\left[j_{i k}(\alpha)-\widetilde{j}_{i k}\right]^{2}
$$

is defined and is continuous in $I=[0+\varepsilon, \pi / 2-\varepsilon]$. Then, by Weierstrass' theorem, $F$ has a minimum in $I$.

3.1. Numerical Examples. Here we give some examples which show numerical results obtained in the reconstruction of the 
TABLE 1

\begin{tabular}{ccccccc}
\hline$i$ & $\lambda_{i}$ & $\gamma_{i}$ & $\eta_{i}$ & $\widehat{a}_{i}$ & $\widehat{b}_{i}$ & $e_{\lambda_{i}} \times 10^{-14}$ \\
\hline 1 & 0.0681 & $\mathbf{0 . 5 8 5 8}$ & 0.1522 & 2.0000 & 1.0000 & 0.4073 \\
2 & 0.2679 & $\mathbf{2 . 0 0 0 0}$ & $\mathbf{0 . 5 8 5 8}$ & 2.0000 & 1.0000 & 0.0622 \\
3 & $\mathbf{0 . 5 8 5 8}$ & $\mathbf{3 . 4 1 4 2}$ & 1.2346 & 2.0000 & 1.0748 & 0.0379 \\
4 & 1.0000 & & $\mathbf{2 . 0 0 0 0}$ & 2.0000 & 0.9191 & 0.0111 \\
5 & 1.4824 & & 2.7654 & 2.0000 & 1.0000 & 0.0599 \\
6 & $\mathbf{2 . 0 0 0 0}$ & & $\mathbf{3 . 4 1 4 2}$ & 2.0000 & 1.0000 & 0.0333 \\
7 & 2.5176 & & 3.8478 & 2.0000 & 1.0880 & 0.0353 \\
8 & 3.0000 & & & 2.0000 & 0.9035 & 0.0148 \\
9 & $\mathbf{3 . 4 1 4 2}$ & & & 2.0000 & 1.0000 & 0.0390 \\
10 & 3.7321 & & & 2.0000 & 1.0000 & 0.0119 \\
11 & 3.9319 & & & 2.0000 & & 0.0113 \\
\hline
\end{tabular}

TABLE 2

\begin{tabular}{lcccccc}
\hline$n$ & $r$ & $p$ & $\alpha$ & $e_{a}$ & $e_{b}$ & $e_{\lambda}$ \\
\hline 9 & 4 & 4 & 0.3 & -14.5655 & -0.6192 & -15.5341 \\
20 & 5 & 14 & 0.6 & -13.9291 & -2.0497 & -15.4525 \\
31 & 11 & 19 & 0.7 & -13.6363 & -2.1453 & -15.4552 \\
41 & 33 & 7 & 1.2 & -13.3794 & -1.9747 & -15.3817 \\
50 & 17 & 32 & 0.8 & -13.3984 & -1.7963 & -15.5074 \\
63 & 27 & 35 & 0.5 & -13.2271 & -1.6714 & -15.3403 \\
71 & 55 & 15 & 1.3 & -12.9105 & -1.4487 & -15.2575 \\
85 & 49 & 35 & 1.1 & -13.1134 & -1.9090 & -15.4762 \\
93 & 37 & 55 & 0.4 & -12.9884 & -1.8209 & -15.4001 \\
101 & 77 & 23 & 0.9 & -12.7881 & -1.9179 & -15.1940 \\
\hline
\end{tabular}

$J$ matrix. In all examples, the reconstructed matrix is the wellknown matrix

$$
J=\left(\begin{array}{ccccc}
2 & 1 & & & \\
1 & 2 & 1 & & \\
& \ddots & \ddots & \ddots & \\
& & 1 & 2 & 1 \\
& & & 1 & 2
\end{array}\right)
$$

which has eigenvalues $\lambda_{i}=2-2 \cos (\pi i /(n+1))$. Moreover, it is also known that if we delete the $(r+1)$ th row and column of $J$, the eigenvalues of the submatrices $J_{r}$ and $J_{p}$ are, respectively, $\gamma_{i}=2-2 \cos (\pi i /(r+1))$ and $\eta_{i}=2-2 \cos (\pi i /(p+1))$, with $p=n-r-1$.

Example 1. In Table 1, we show the results associated with the reconstructed matrix $\widehat{J}$, of the form (44), with $n=11, r=3$, and $p=7$ for $\alpha=0.5$. The given eigenvalues are shown in the second, third, and fourth column. In the fifth and sixth column we list the diagonal and codiagonal entries of $\widehat{J}$. In the last column, we show the relative error $e_{\lambda_{i}}=\left\|\lambda_{i}-\hat{\lambda}_{i}\right\|_{2} /\left\|\lambda_{i}\right\|_{2}$ with respect to the exact eigenvalues $\lambda_{i}$ of the matrix $J$ and the eigenvalues $\hat{\lambda}_{i}$ of $\widehat{J}$.

Example 2. In Table 2, we show the results associated with the reconstructed matrix $\widehat{J}$, by considering appropriate orders of $\widehat{J}$ and for arbitrary values of $\alpha$, listed from the first to fourth
TABLE 3

\begin{tabular}{lcccccc}
\hline$n$ & $r$ & $p$ & $\alpha_{\mathrm{opt}}$ & $e_{a}$ & $e_{b}$ & $e_{\lambda}$ \\
\hline 9 & 4 & 4 & 0.7854 & -14.6213 & -15.4519 & -15.4589 \\
20 & 5 & 14 & 0.5639 & -13.9393 & -13.8169 & -15.5854 \\
31 & 11 & 19 & 0.6591 & -13.7277 & -13.5305 & -15.5647 \\
41 & 33 & 7 & 1.1192 & -13.2729 & -14.1176 & -15.2605 \\
50 & 17 & 32 & 0.6361 & -13.2659 & -14.2862 & -15.2269 \\
63 & 27 & 35 & 0.7227 & -13.2019 & -14.3889 & -15.2199 \\
71 & 55 & 15 & 1.0799 & -13.0260 & -14.3109 & -15.1878 \\
85 & 49 & 35 & 0.8672 & -12.9445 & -14.1855 & -15.1569 \\
93 & 37 & 55 & 0.6891 & -12.8941 & -14.2323 & -15.2115 \\
101 & 77 & 23 & 1.0644 & -12.8154 & -14.0673 & -15.0930 \\
\hline & & & & & &
\end{tabular}

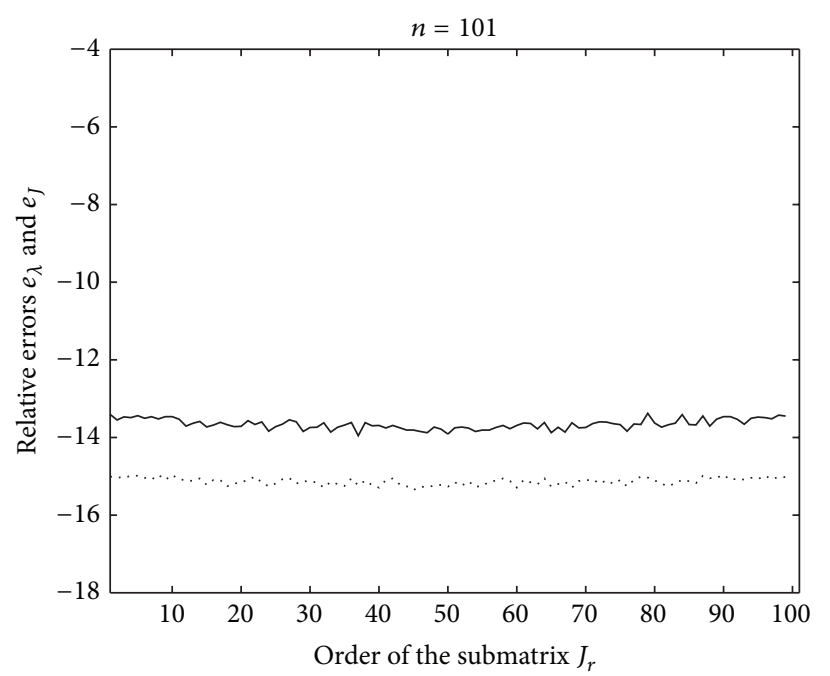

$$
-e_{J}
$$

FIgURE 4: 69 of 90 reconstructions of $J$ with identical eigenvalues.

column. The relative errors $e_{a}=\log \left(\|a-\hat{a}\|_{2} /\|a\|_{2}\right)$ and $e_{b}=$ $\log \left(\|b-\widehat{b}\|_{2} /\|b\|_{2}\right)$, with respect to the diagonal and codiagonal entries of $J$ and $\widehat{J}$, respectively, are shown in the fifth and sixth column. In the last column, we present the relative errors $e_{\lambda}=$ $\log \left(\|\lambda-\widehat{\lambda}\|_{2} /\|\lambda\|_{2}\right)$, defined as in Example 1 .

Example 3. In the reconstructed matrix $\widehat{J}$, by considering the same orders of Example 2, we add an optimization process of Golden Section Search [11], of parameter $\alpha$, obtaining an optimal $\alpha$, denoted as $\alpha_{\text {opt }}$ and listed in the fourth column of Table 3. In the last three columns the relative errors $e_{a}, e_{b}$, and $e_{\lambda}$ are shown.

Example 4. In this example, we reconstruct the matrix $\widehat{J}$ for $n=101$ and 503. In each case we do as many reconstructions of $\widehat{J}$ as values $r$ can take. The values of $n$ allow us to have various reconstructions with $\sigma(J) \cap \sigma\left(J_{r}\right) \cap \sigma\left(J_{p}\right) \neq \emptyset$. Figures 4 and 5 show the plots of the relative errors $e_{J}=\log \left(\|J-\widehat{J}\|_{2} /\|J\|_{2}\right)$ and $e_{\lambda}=\log \left(\|\lambda-\widehat{\lambda}\|_{2} /\|\lambda\|_{2}\right)$. 


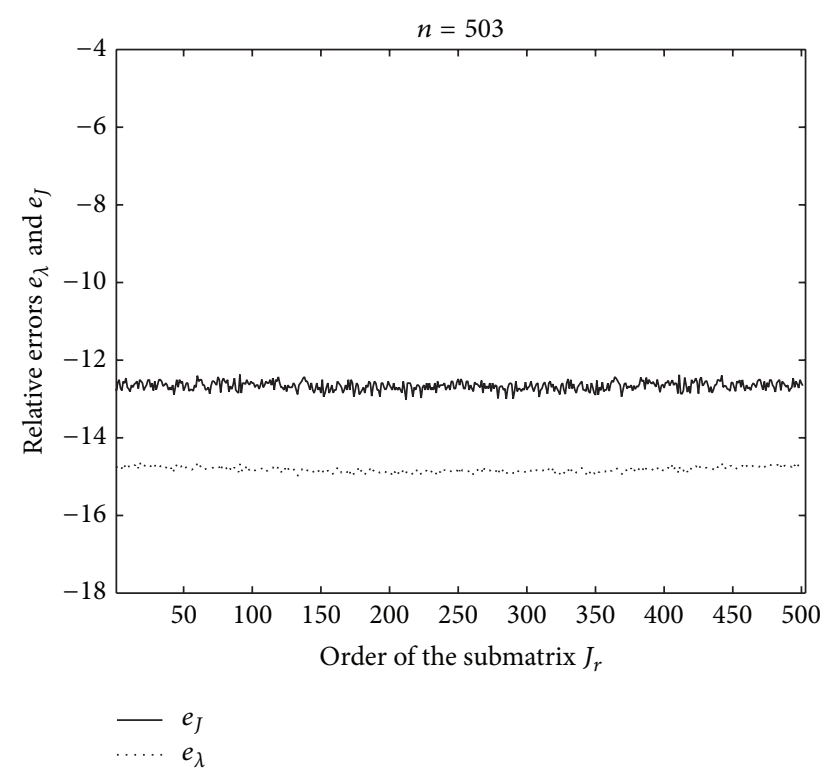

FIGURE 5: 359 of 501 reconstructions of $J$ identical eigenvalues.

The results of our numerical experiments confirm that our method works quite well.

\section{Conflict of Interests}

The authors declare that there is no conflict of interests regarding the publication of this paper.

\section{Acknowledgments}

This paper was supported by Project UTA 4730-13, Chile, and Universidad Católica del Norte, Chile.

\section{References}

[1] B. N. Datta, Numerical Linear Algebra and Applications, SIAM, Philadelphia, Pa, USA, 2nd edition, 2009.

[2] B. N. Datta, Numerical Methods for Linear Control Systems Design and Analysis, Elsevier/Academic Press, New York, NY, USA, 2003.

[3] B. N. Datta and D. R. Sarkissian, "Theory and computations of some inverse eigenvalue problems for the quadratic pencil," in Structured Matrices in Operator Theory, Control, and Signal and Image Processing, vol. 280 of Contemporary Mathematics, pp. 221-240, American Mathematical Society, Providence, RI, USA, 2001.

[4] G. M. L. Gladwell, Inverse Problems in Vibration, Martinus Nijhoff, Dordrecht, The Netherlands, 2004.

[5] Y. M. Ram and S. Elhay, "Constructing the shape of a rod from eigenvalues," Communications in Numerical Methods in Engineering, vol. 14, no. 7, pp. 597-608, 1998.

[6] G. H. Golub and C. F. van Loan, Matrix Computations, vol. 3 of Johns Hopkins Series in the Mathematical Sciences, Johns Hopkins University Press, Baltimore, Md, USA, 2nd edition, 1989.
[7] J. C. Egaña and R. L. Soto, "On the numerical reconstruction of a spring-mass system from its natural frequencies," Proyecciones, vol. 19, no. 1, pp. 27-41, 2000.

[8] G. M. L. Gladwell and N. B. Willms, "The reconstruction of a tridiagonal system from its frequency response at an interior point," Inverse Problems, vol. 4, no. 4, pp. 1013-1024, 1988.

[9] W. B. Gragg and W. J. Harrod, "The numerically stable reconstruction of Jacobi matrices from spectral data," Numerische Mathematik, vol. 44, no. 3, pp. 317-335, 1984.

[10] E. R. Jessup, "A case against a divide and conquer approach to the nonsymmetric eigenvalue problem," Applied Numerical Mathematics, vol. 12, no. 5, pp. 403-420, 1993.

[11] W. H. Press, S. A. Teukolsky, W. T. Vetterling, and B. P. Flannery, Numerical Recipes, Cambridge University Press, 3rd edition, 2007. 


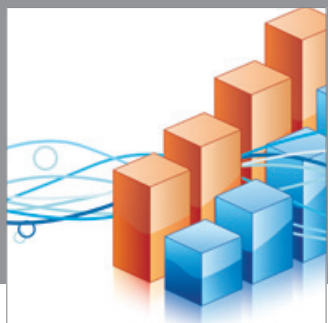

Advances in

Operations Research

mansans

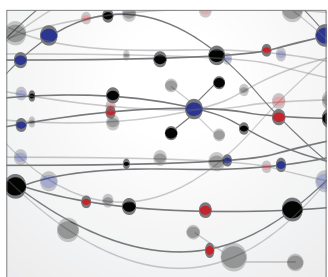

The Scientific World Journal
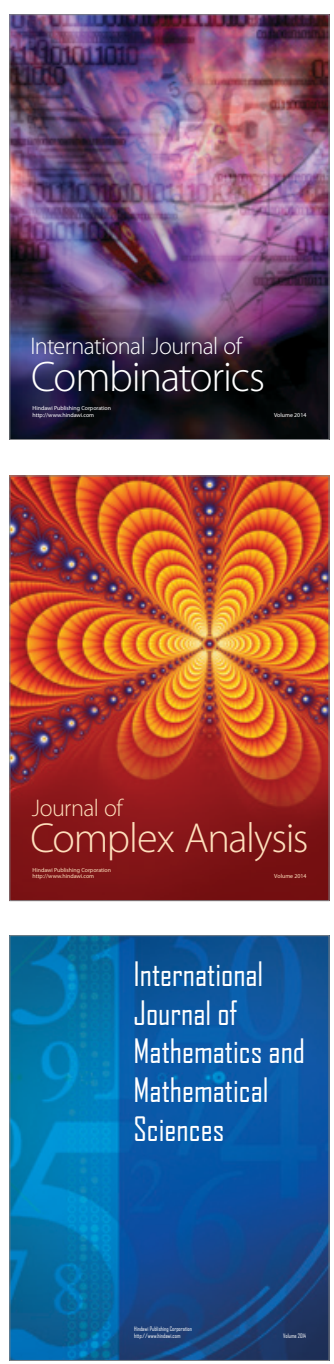
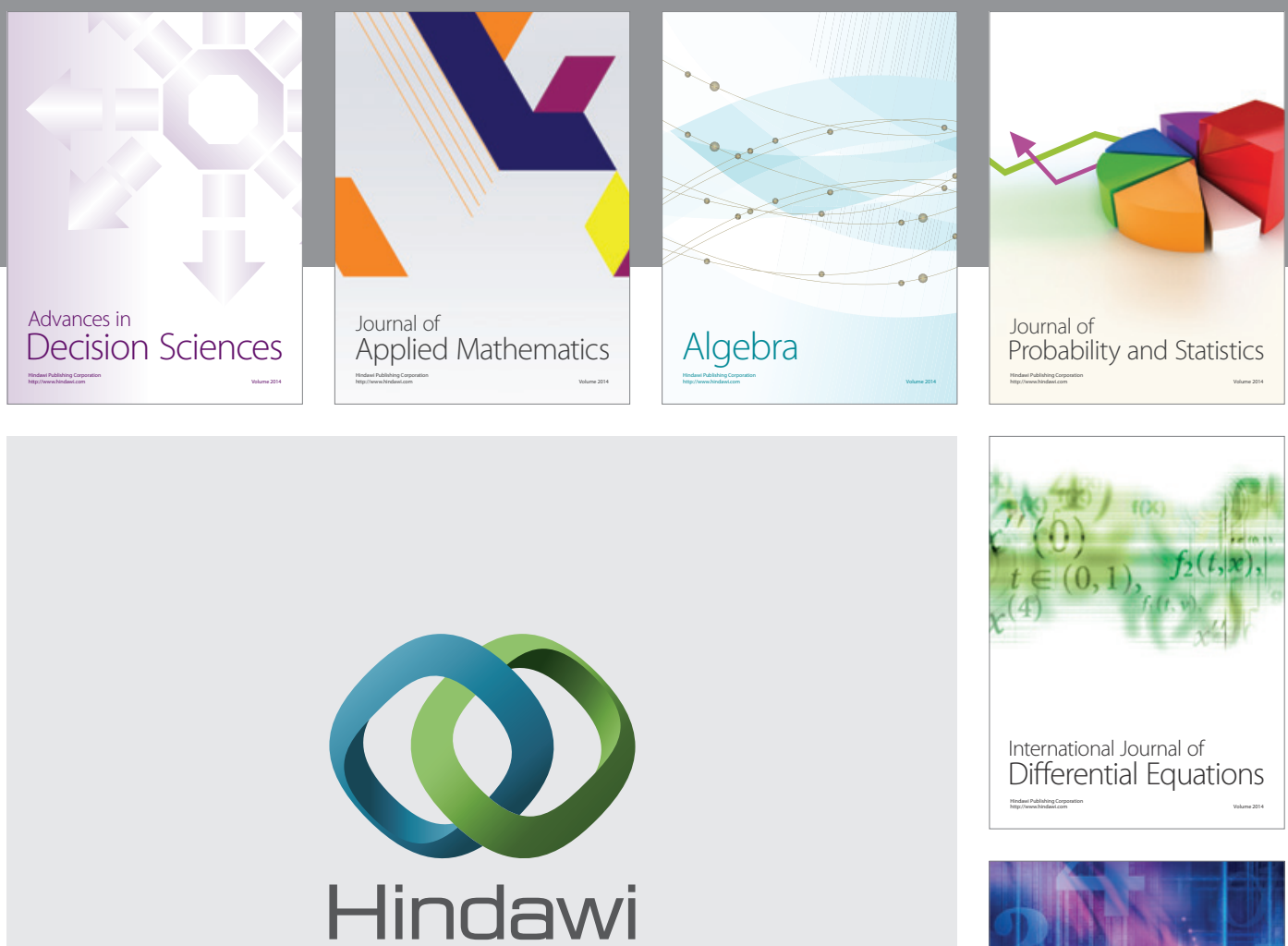

Submit your manuscripts at http://www.hindawi.com
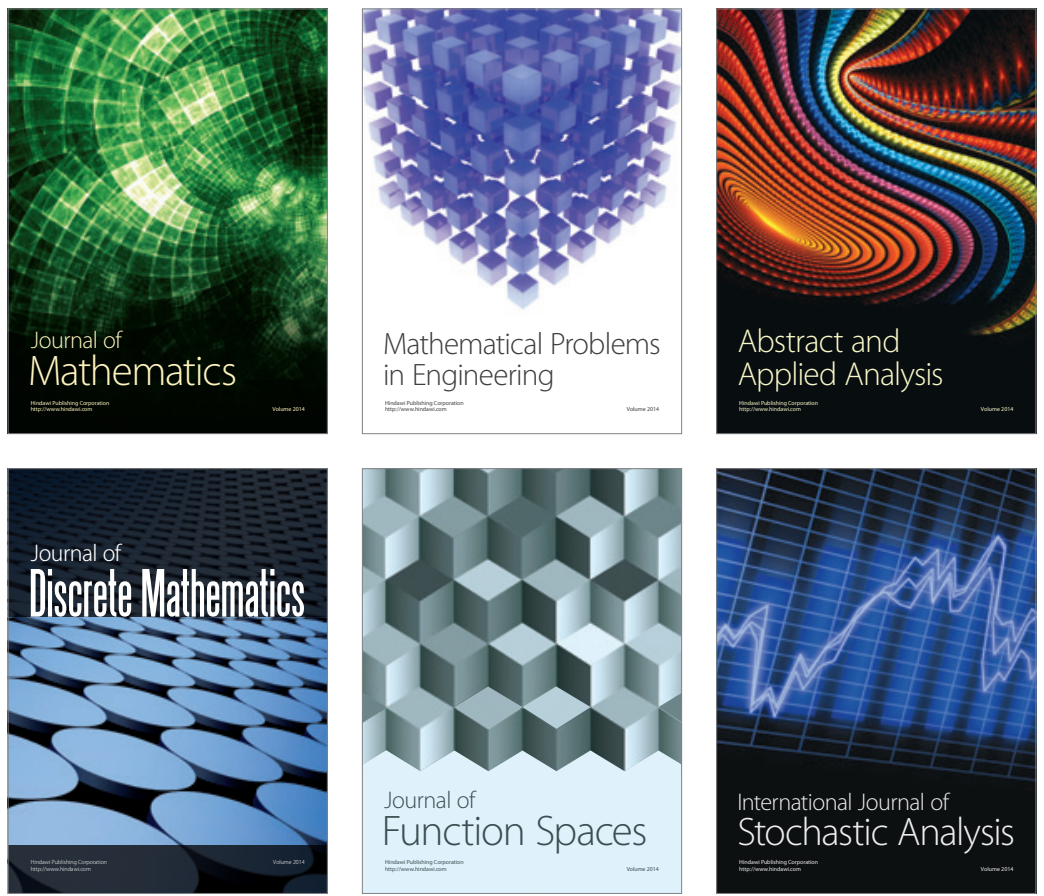

Journal of

Function Spaces

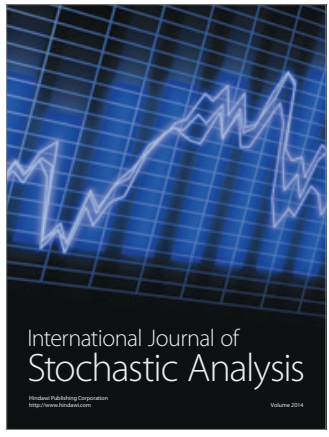

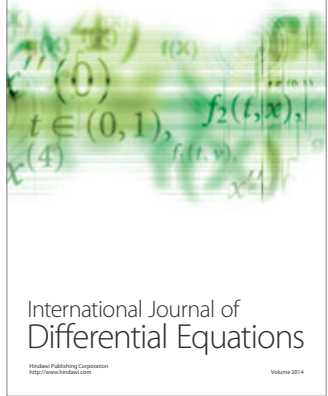
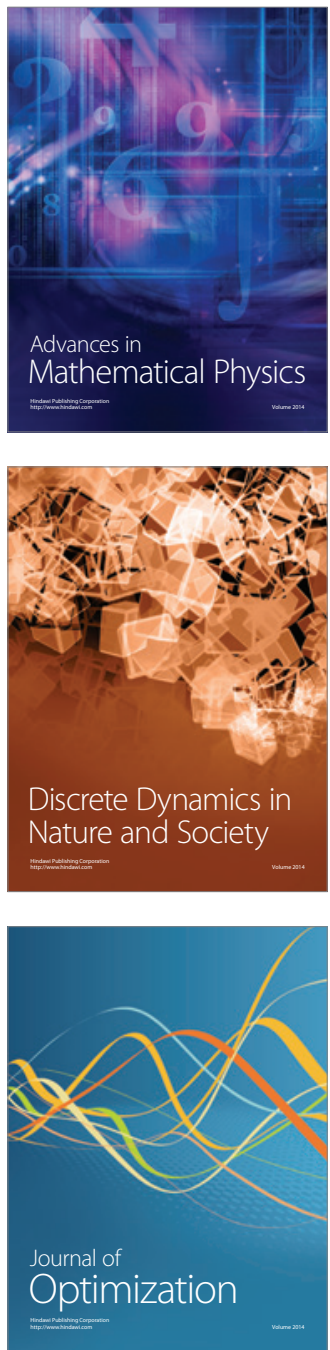